\title{
Effective transcutaneous immunization by antigen-loaded flexible liposome in vivo
}

This article was published in the following Dove Press journal:

International Journal of Nanomedicine

7 December 2011

Number of times this article has been viewed

$\mathrm{Ni} \mathrm{Li}$

Li-Hua Peng'

Xi Chen'

Shinsaku Nakagawa ${ }^{2}$

Jian-Qing Gao'

'Institute of Pharmaceutics, College

of Pharmaceutical Sciences,

Zhejiang University, Hangzhou,

Zhejiang, People's Republic of China;

${ }^{2}$ Department of Biotechnology and

Therapeutics, Graduate School

of Pharmaceutical Sciences,

Osaka University, Suita, Japan
Correspondence: Jian-Qing Gao Institute of Pharmaceutics, College of Pharmaceutical Sciences, Zhejiang University, People's Republic of China $\mathrm{Tel} / \mathrm{fax}+8657$ I 88208437

Email gaojianqing@zju.edu.cn
Background: Transcutaneous vaccines have received wide attention due to their easy-to-use, needle-free, noninvasive delivery. However, the novel barrier function of stratum corneum hinders the transport of antigen and adjuvant in transcutaneous immunization. Novel nanoscale delivery systems employing, for example, liposomes and nanoparticles, have been widely investigated to overcome the penetration barrier of stratum corneum for effective transcutaneous immunization.

Objective: The objective of this study was to prepare two types of flexible liposomes and determine their efficacies for the transcutaneous delivery of antigen and the subsequent immune response induced in vivo.

Methods: Ovalbumin (OVA) liposome-based transcutaneous vaccines were prepared using reverse-phase evaporation and film-dispersion methods. Particle sizes and antigen encapsulating efficiency were then evaluated. After application to bare mouse skin, topical sites were examined for the presence of fluorescence-labeled liposome. The efficacy of the transcutaneously delivered OVA-loaded flexible liposome in activating the immune responses was investigated by detecting serum immunoglobulin $\mathrm{G}$ levels. The influence of an adjuvant, imiquimod, in the transcutaneous immunization was also tested.

Results: Two flexible liposomes with well-encapsulated OVA were successfully prepared by film-dispersion or reverse-phase evaporation methods. The sizes of the prepared flexible liposomes ranged from 200 to $400 \mathrm{~nm}$. In vivo, the fluorescence-labeled liposome was detected in hair-follicle ducts, indicating that the flexible liposome can penetrate the skin barrier through the hair follicles. Upon transcutaneous administration, the OVA-encapsulated flexible liposome elicited a strong immune response similar to that of positive control (ie, OVA solution administrated by subcutaneous injection with $\mathrm{Al}(\mathrm{OH})_{3}$ as an adjuvant). Co-administration of imiquimod with the OVA-loaded liposome expressed a significant enhancement on the transcutaneous immune responses.

Conclusion: Results of this study highlight the nanoscale formulation, flexible liposome, as a promising carrier for the transcutaneous delivery of antigen proteins. Imiquimod was shown to be an effective adjuvant as a transcutaneous immunization enhancer with the potential for transcutaneous vaccine development.

Keywords: flexible liposome, transcutaneous vaccine, immunization enhancement, adjuvant

\section{Introduction}

As a biological preparation that improves immunity to a particular disease, vaccine is one of the most important accomplishments in medicine. Vaccines based on active peptides and proteins have been developed as a consequence of the progress of biotechnological techniques and genetic engineering. However, while most vaccines 
administered via injection are effective, the problems of pain, needle-related infections or injuries, the need for trained personnel, and storage or transport issues remain. ${ }^{1}$ The practice of reusing needles causes the death of at least 1.3 million people per year from hepatitis B and acquired immune deficiency syndrome, therefore the desire to eliminate hypodermic needles greatly motivates the development of needle-free vaccines. ${ }^{2}$ Transcutaneous immunization is a new method that utilizes topical application of an adjuvant and vaccine antigen to intact skin for vaccination. ${ }^{3}$ In this strategy, antigen with novel formulations is delivered by transporting the antigen to the epidermis. The antigen is then taken up by Langerhans cells and migrates to lymph nodes ${ }^{4}$ to induce an immune response. Transcutaneous immunization by topical drug-delivery systems incorporating antigens avoids direct contact between potent adjuvants (sometimes slightly toxic) and the general circulation, hence offering the advantages of rapid distribution, self-administration, and enhanced safety. ${ }^{5,6}$ Due to its easy-to-use, needle-free, and noninvasive features, transcutaneous immunization represents an attractive alternative to vaccine delivery via subcutaneous and intramuscular routes. ${ }^{7}$

For example, the authors have recently reported a hydrogel patch formulation that was shown to promote the penetration of antigenic proteins into stratum corneum to induce a strong immune response. ${ }^{8,9}$ However, transcutaneous vaccine has not been exploited to its full potential due to the inherent barrier function of stratum corneum. As it is not easy for large proteins and viral particles to penetrate through stratum corneum under natural conditions, the main challenge for transcutaneous immunization is thus to enhance the crossover of antigens through the epidermis and initiate physical effective immunogenicity. Currently, in addition to disrupting the skin barrier by removal of stratum corneum, increasing efforts have been put into developing nanometer drug-delivery systems. For example, nanoparticles and transfersomes achieve transcutaneous immunization enhancement by stimulating skin penetration and protecting the antigen from degradation, which enables uptake by more antigenpresenting cells (APCs). In the authors' previous studies, nanoscaled transfersomes ${ }^{10-13}$ and nanoparticles ${ }^{14-17}$ have been shown to be excellent carriers capable of delivering antigenic proteins to APCs. They elicit potent immune responses based on antigen-specific cytotoxic $\mathrm{T}$ lymphocytes and promote the migration of the antigen-capturing APCs to regional lymph nodes via subcutaneous injection or nasal cavity. Among them, the novel vesicular carrier system known as transfersomes, composed of phospholipid, surfactant, and water, proved to be effective for transdermal delivery enhancement. With a similar structure to biological membranes, the transfersomal system, which includes deformable liposomes and noisomes, have been formulated for the topical/transdermal delivery of bioactive compounds, for example, antibiotics, proteins, and nucleic acids for skin penetration. The frequency of using transcutaneous protein delivery by means of transfersomes is reported to be increasing. ${ }^{18}$ Methods have been used for the encapsulation of peptides and proteins in liposomes. Currently, several methods have been developed to form unilamellar vesicles from multibilayers, ${ }^{19}$ for example, the film-dispersion, ${ }^{20}$ reverse-phase evaporation, ${ }^{21}$ and dehydration-rehydration methods. ${ }^{22}$ Furthermore, to increase liposome flexibility, ethanol and the nonionic surfactant polysorbate 80 could be incorporated for the preparation of flexible liposomes (FLs), which contain phosphatidylcholine and a surfactant, and consists of at least one inner aqueous compartment surrounded by a lipid bilayer. When mixed with stratum corneum lipids, FLs can carry a remarkable amount of lipid mass into the skin and therefore be advantageous in promoting the cutaneous drug disposition after disrupting the skin barrier with its flexible bilayer. ${ }^{23}$ It has been reported that FLs could stimulate transcutaneous immune response by acting as an adjuvant. ${ }^{24}$ Furthermore, besides the physical and pharmaceutical methods, adjuvants are frequently used in immunology to stimulate the immune system to increase the response to a vaccine. The combination of adjuvant and specific vaccine antigen can accelerate, prolong, or enhance antigen-specific immune responses. The range of adjuvant candidates is wide. The colloidal aluminum hydroxide and Freund's adjuvant are commonly used candidates. However, not all of these adjuvants are effective in the transcutaneous immunization due to their relatively large sizes, which do not favor skin penetration. In this study, a fluorescent material, fluorescein sodium $(\mathrm{NaFl})$, was chosen to prepare FLs along with a suitable amount of sodium deoxycholate as a surfactant. It was found that this FL could enhance drug content in the skin. Secondly, the ratio of phospholipids $\mathrm{S}$ 100 and cholesterol, types of organic solvent, and the amount of ovalbumin (OVA) were optimized when the drug was changed from $\mathrm{NaFl}$ to OVA. The optimizations were achieved by characterization of particle size, entrapment efficiency, and stability. FLs were first prepared with the optimized film-dispersion and reversed-phase evaporation methods and characterized in vitro. The entrapment efficiency and release properties of the FLs for a model antigen, OVA, were then evaluated. Later, transcutaneous OVA delivery, transferred by the FLs, was studied before the investigation of in vivo 
transcutaneous immunization. Additionally, several other immunogenic adjuvants were evaluated for their applications in transcutaneous immunization and their influence on the efficacy of OVA-FLs vaccine.

\section{Materials and methods Materials}

Phospholipid S 100 was obtained from Avanti Polar Lipids (Alabaster, AL). OVA and cholesterol were obtained from Sigma-Aldrich (St Louis, MO). Sephadex G-100 was obtained from Beijing Henghui Science and Technology Development (Beijing, China). NaFl, Tween ${ }^{\circledR} 20$, and sodium deoxycholate were from Aladdin (Shanghai, China) and Imiquimod was from Dinghui Chemical (Wuhan, China). BCA kit was from Nanjing BiYunTian kits biotechnology company (Jiangsu, China). Horseradish peroxidase conjugated goat antimouse IgG was obtained from Wuhan Boster (Wuhan, China). Fluorescein isothiocyanate-conjugated OVA was acquired from Beijing Boaosen biotechnology (Beijing, China). All reagents were of analytic grade and used as received without further purification.

\section{Instruments}

Malvern Zetasizer (Zeta Master; Malvern Instruments Ltd, Malvern, UK) and a microplate enzyme-linked immunosorbent assay reader KHB ST-360 (an automatic microplate reader, Shanghai Zhihua Medical Instrument, China).

\section{Methods}

\section{Preparation of common liposomes (CLs) by film-dispersion method}

To prepare CLs using this method, $200 \mathrm{mg}$ phospholipids $\mathrm{S} 100$ and $50 \mathrm{mg}$ cholesterol were dissolved in $15 \mathrm{~mL}$ chloroform, dried under reduced pressure in a round-bottom glass flask and stored in vacuum for at least 1 hour, in order to make a very thin film. The lipid film was then hydrated with $5 \mathrm{~mL}$ of distilled water using $8 \mathrm{mg} \mathrm{NaFl}$. Later, $5 \mathrm{~mL}$ phosphate-buffered saline (PBS) solution was added. After hydration of the film, large vesicles arose, entrapping solvent and solutes in their aqueous core and between lamellae. The suspensions were vortexed gently for 2-3 hours, followed by sonication for 30 minutes.

\section{Preparation of CLs by reverse-phase evaporation method}

With the reverse-phase evaporation dispersion method, $200 \mathrm{mg}$ phospholipids S 100 and $50 \mathrm{mg}$ cholesterol were dissolved in
$15 \mathrm{~mL}$ chloroform. Later, $8 \mathrm{mg} \mathrm{NaFl}$ diluted in $5 \mathrm{~mL}$ distilled water was added. Using probe sonication (400 W, 2 minutes), the $\mathrm{w} / \mathrm{o}$ emulsions were formed and dried under reduced pressure for 15 minutes in a round-bottom glass flask. After solvent evaporation, the compartments were collapsed to give a gel, $5 \mathrm{~mL}$ PBS solution was added, and the suspensions were gently vortexed under vacuum for at least 3 hours.

\section{Preparation of FLs by film-dispersion method}

FLs were prepared in this way by dissolving $200 \mathrm{mg}$ phospholipids S 100 and $50 \mathrm{mg}$ cholesterol in $15 \mathrm{~mL}$ chloroform. This was dried under reduced pressure in a round-bottom glass flask and stored in vacuum for at least 1 hour to make a very thin film. The lipid film was then hydrated with $5 \mathrm{~mL}$ of distilled water with 25 mg OVA (A5503; Sigma-Aldrich). Later, $30 \mathrm{mg}$ sodium deoxycholate diluted in $5 \mathrm{~mL}$ PBS solution was added. Large vesicles formed after hydration of the film, entrapping solvent and solutes in their aqueous core and between lamellae. The suspensions were vortexed gently for 2-3 hours followed by sonication for 30 minutes.

\section{Preparation of FLs by reverse-phase evaporation method}

With the reverse-phase evaporation dispersion method, $200 \mathrm{mg}$ phospholipids S 100 and $50 \mathrm{mg}$ cholesterol were dissolved in $15 \mathrm{~mL}$ chloroform. After this, $25 \mathrm{mg}$ OVA diluted in $5 \mathrm{~mL}$ distilled water was added. Using probe sonication ( $400 \mathrm{~W}, 2$ minutes), the w/o emulsions formed were dried under reduced pressure for 15 minutes in a round-bottom glass flask. After solvent evaporation, the compartments were collapsed to give rise to a gel, $30 \mathrm{mg}$ sodium deoxycholate diluted in $5 \mathrm{~mL}$ PBS solution was added, and the suspensions were gently vortexed under vacuum for at least 3 hours.

\section{In vitro diffusion studies of different $\mathrm{NaFl}$ formulations}

To determine the distribution of different $\mathrm{NaFl}$ formulations in the stratum corneum and viable skin, in vitro diffusion studies were carried out. The pig skin used was prepared from full thickness tissue, which had been cleaned of underlying fat then mounted in Franz-type diffusion cells with an exposed surface area of approximately $2.83 \mathrm{~cm}^{2}$ and receptor phase volume of roughly $6.8 \mathrm{~mL}$ saline. One milliliter of each prepared $\mathrm{NaFl}$ formulation was added in the supply phase. The receptor phase was continuously stirred with magnetic fleas and maintained in a water bath at $37^{\circ} \mathrm{C}$ to give a skin 
surface temperature of $\sim 32^{\circ} \mathrm{C}$. The $\mathrm{NaFl}$ formulations were divided into the following groups:

- Solution: NaFl solution

- CLs (reverse): CLs prepared by reverse-phase evaporation method

- CLs (film): CLs prepared by film-dispersion method

- FLs (reverse): FLs prepared by reverse-phase evaporation method

- FLs (film): FLs prepared by film-dispersion method.

After 8 hours, the skins were washed with saline. The distribution of $\mathrm{NaFl}$ in the skin was directly assessed using adhesive tape (Scotch ${ }^{\mathrm{TM}}$; 3M Co, Two Harbors, MN), using the tape-stripping method. Tape stripping was performed 30 times on the surface of the skin. Each strip was removed with one quick movement. The stripped tapes $(\mathrm{NaFl}$ in stratum corneum) were put in a beaker and the $\mathrm{NaFl}$ was separated from the tape using 10\% isopropanol diluted in saline. Any residual $\mathrm{NaFl}$ left in the viable skin after tape stripping was also extracted. The viable skin was cut into pieces, diluted in $5 \mathrm{~mL}$ saline mixture, sonicated for 1 hour, and centrifuged to extract the supernatant. Saline $(5 \mathrm{~mL})$ was added to the pellet after centrifugation, and then ultrasound and centrifuged to extract the supernatant; the two supernatants were combined together for determination. The $\mathrm{NaFl}$ content in the stratum corneum and viable skin were determined by fluorescence spectrophotometer.

\section{Characterization of OVA-FLs}

The structure and surface characteristics of the prepared liposome systems were viewed under transmission electron microscope (JEM-1230; JEOL, Tokyo, Japan). A drop of liposome dispersion was diluted with deionized water. A drop of the diluted liposome dispersion was then applied onto a carbon coated 300 mesh copper grid and left for 1 minute to allow the adherence between liposome and the carbon substrate. Excess of dispersion was removed by blotting the drop with filter paper. Using $1 \% \mathrm{w} / \mathrm{v}$ phosphotungstic acid solution for negative staining, the grid was kept in phosphotungstic acid solution for 2 minutes and observed under transmission electron microscope under wet conditions. Photographs were taken to reveal morphological details of vesicular assemblages. The FLs were also characterized for their sizes, PDI, and zeta potential with a dynamic light-scattering method, in a multimodal mode using a computerized inspection system (Zeta Master; Malvern Instruments Ltd, Malvern, UK). For size measurement of vesicles, vesicular suspension was mixed with the appropriate medium (PBS pH 6.5) and measurements were conducted in triplicate.

\section{Antigen entrapment efficiency of FLs}

Entrapment efficiency of the OVA in FLs was determined after separating free OVA from the liposomes by molecular exclusion chromatography using a Sephadex G-100 minicolumn. Briefly, OVA-loaded liposomes were suspended in $5 \mathrm{~mL}$ water and put into a space of Sephadex G-100 minicolumn centrifugation, which was spun to separate the free OVA from liposome dispersions. The unentrapped antigen was removed after centrifugation, together with the Sephadex G-100 minicolumn. Free OVA medium $(20 \mu \mathrm{L})$ was taken for OVA measurement by standardized bicinchoninic acid (BCA) measurement method. Entrapment efficiency was defined as the ratio of liposome-associated OVA to total OVA used. The experiments were performed in triplicate.

\section{OVA stability in FLs}

For the characterization of antigen bioactivity, the integrity of OVA under nondenaturing condition was identified through sodium dodecyl sulfate polyacrylamide gel electrophoresis (SDS-PAGE) under reducing conditions. OVA was identified through SDS-PAGE under reducing conditions using a known concentration of OVA in 5\% stacking gel and $15 \%$ separation (resolving) gel (Bio-Rad Laboratories, Inc, Richmond, CA). Protein bands were detected by Coomassie Brilliant Blue staining. In the presence of the strong reducing agent sodium dodecyl sulfate (SDS) and heat, the proteins were dissociated before they were applied on the gel. SDS-PAGE experiments were performed on 5\% stacking gel and $8 \%$ resolving gel following the standardized protocol. The samples were heated to $95^{\circ} \mathrm{C}$ for 5 minutes prior to running. After electrophoresis, the gel with samples was dried.

\section{In vitro release of OVA from FLs}

Briefly, $5 \mathrm{~mL}$ of the OVA-loaded liposome dispersions after the Sephadex G-100 minicolumn centrifugation was transferred into a large tube containing $30 \mathrm{~mL}$ phosphate buffer (pH 7.4) in thermostat oscillators at $37^{\circ} \mathrm{C} \pm 1^{\circ} \mathrm{C}$ and mechanically shaken at a suitable rotation speed. At designated time intervals, $0.5 \mathrm{~mL}$ sample of the dialysis medium was taken for BCA protein measurement of OVA and the same volume of fresh medium was then added. The above $0.5 \mathrm{ml}$ samples were centrifuged at a speed of 20,000 rpm to separate the released OVA (supernatant) and the intact liposome dispersions (pellet). The concentration of supernatant was measured for the released OVA using a BCA kit. The protocol of the BCA kit is similar to the aforementioned method used to determine the antigen entrapment efficiency of FLs. The release experiments were performed in triplicate. 


\section{Transcutaneous delivery of OVA-FLs in vivo}

Female BALB/c mice 6-8 weeks old (18-20 g) were used in all experiments. All animal experiments were conducted in accordance with the national regulations for care and use of laboratory animals. Mice were anesthetized with pentobarbital sodium. Most of the hair from the abdominal part of each mouse was shaved. Neither cut nor skin irritation was observed. The shaved area was wiped with cotton swab. Liposome formulations loaded with fluorescein isothiocyanate-conjugated OVA (FITC-OVA) were uniformly applied onto the shaved area $(2 \times 2 \mathrm{~cm})$, using a pipette tip, for 30 minutes. Mice were then sacrificed and their backs were washed twice with water and dried with a cotton swab. Skin samples of $1 \times 1 \mathrm{~cm}$ from the treated areas were removed and frozen. Sections $6 \mu \mathrm{m}$ thick were obtained using a Leica CM 1500 (Leica Biosystems GmbH, Wetzlar, Germany) cryotome and collected on a glass slide. Fluorescence microscopy was used for detection. The fluorescent liposomes produced green signals.

\section{Transcutaneous immunization of OVA-FLs in vivo}

Groups of seven mice were immunized by topical application of different OVA formulations (each containing $200 \mu \mathrm{g}$ OVA). Mice were shaved and various antigen formulations were applied as described. After 1 hour, the shaved area was washed with water and dried with a cotton swab. Imiquimod ( $5 \mu \mathrm{g}$ /mouse) was included in certain formulations as adjuvant. An equal booster dose of each formulation was administered by the same route fortnightly. The following groups were included in the study:

- Group 1: liposome 1 (prepared by film-dispersion method) with or without adjuvant imiquimod by transcutaneous immunization.

- Group 2: liposome 1 (prepared by film-dispersion method) applied on intact skin or pretreated skin where stratum corneum was removed transcutaneously; imiquimod was applied as an adjuvant.

- Group 3: liposome 1 (prepared by film-dispersion method) or liposome 2 (prepared by reverse-phase evaporation method) applied on pretreated skin with stratum corneum removed transcutaneously; imiquimod was applied as an adjuvant.

- Group 4: liposome 1 (prepared by film-dispersion method) applied transcutaneously on intact skin, with the adjuvant imiquimod; positive control mice were subcutaneously injected with $200 \mu \mathrm{g}$ OVA solution with $\mathrm{Al}(\mathrm{OH})_{3}$ as adjuvant.
Blood samples were collected fortnightly $(2,4,6$, and 8 weeks) after primary immunization.

\section{Determination of specific anti-OVA immunoglobulin (Ig) G antibody levels in the serum by enzyme-linked immunosorbent assay}

One hundred microliters of OVA $(10 \mu \mathrm{g} / \mathrm{mL}$ in phosphate buffer, $\mathrm{pH}$ 7.4) was used to coat each well of a 96-well plate, which was then incubated at $4^{\circ} \mathrm{C}$ overnight. Following this, the plate was washed three times with TTBS $(0.05 \%[\mathrm{v} / \mathrm{v}]$ Tween ${ }^{\circledR} 20$ in tris-buffered saline) to remove unloaded OVA. Fifty microliters of $5 \%$ bovine serum albumin was added into each well and plate was incubated for $2 \mathrm{~h}$ at room temperature and washed three times with TTBS. One hundred microliters of diluted serum sample was added to each well and incubated for another 2 hours at room temperature and later washed three times with TTBS. One hundred microliters of diluted horseradish peroxidase conjugated goat antimouse $\operatorname{IgG}$ was added to each well and incubated for 2 hours. The plate was washed again three times with TTBS and then $100 \mu \mathrm{L}$ of substrate solution (3,3,5,5-tetramethyl benzidine containing hydrogen peroxide) was added to each well. The plate was then incubated in darkness at room temperature for 15 minutes. Adding $50 \mu \mathrm{L}$ of $2 \mathrm{M} \mathrm{H}_{2} \mathrm{SO}_{4}$ to each well terminated the reaction. The absorbance was measured at $450 \mathrm{~nm}$ using a microplate enzyme-linked immunosorbent assay reader (Lab System Multiscan, Finland).

\section{Statistical analysis}

Data were expressed as mean and standard deviation (SD). Statistical significance of the differences between groups was assessed using Student's $t$-test. Analysis of variance was used for comparison of more than two samples. Difference of twotailed $P<0.05$ was considered statistically significant.

\section{Results}

\section{Preparation and characterization of the liposome formulations}

In previous work, the authors chose the fluorescent watersoluble material $\mathrm{NaFl}$ to prepare two types of FLs (data not shown). Determination of NaFl in the skin by transcutaneous delivery revealed that, compared with both the $\mathrm{CL}$ and the $\mathrm{NaFl}$ solution groups, $\mathrm{NaFl}$ contents in the stratum corneum and viable skin were significantly increased in the FL groups (Table 1). In addition, the two FLs with well-encapsulated OVA, either by film-dispersion or reverse-phase evaporation methods, were successfully prepared. The properties of the 
Table I Distribution of different NaFl formulations in the stratum corneum and viable skin

\begin{tabular}{lccccc}
\hline Distribution & Solution & CLs (reverse) & CLs (film) & FLs (reverse) & FLs (film) \\
\hline Stratum corneum & $7.62 \pm 1.84$ & $22.92 \pm 0.87^{* *}$ & $17.53 \pm 4.53^{*}$ & $16.83 \pm 3.15^{*}$ & $8.81 \pm 5.43$ \\
Viable skin & $991.57 \pm 43.67$ & $1980.92 \pm 661.19$ & $628.03 \pm 27.62^{*}$ & $2894.59 \pm 178.79 * *$ & $930.46 \pm 356.25$ \\
\hline
\end{tabular}

Notes: All the formulations were applied by transcutaneous route in the pig skin $\left(\mathrm{ng} / \mathrm{cm}^{2}\right.$, mean $\left.\pm \mathrm{SD}, \mathrm{n}=3\right)$. The statistical significance of the differences between groups was investigated using Student's $t$-test. *Versus solution group, $P<0.05$; **versus solution group, $P<0.01$. Solution: NaFl solution.

Abbreviations: CLs (reverse), common liposomes prepared by reverse-phase evaporation method; CLs (film), common liposomes prepared by film-dispersion method; FLs (reverse), flexible liposomes prepared by reverse-phase evaporation method; FLs (film), flexible liposomes prepared by film-dispersion method; NaFI, fluorescein sodium.

two liposomes were characterized via vesicle size and size distribution with a Malvern Zetasizer (Figure 1A). Their structure and surface characteristics were measured using a transmission electron microscope (Figure 1B). According to the transmission electron microscope images, the discrete liposomes 1 and 2 were spherical and their round shapes would be maintained with homogeneous structures. The liposomes were measured at 200-400 nm. With BCA protein assays, the OVA entrapment efficiency of liposome 1 and liposome 2 were determined as $47.9 \% \pm 8.9 \%$ and $77.9 \% \pm 19.2 \%$, respectively.

\section{OVA stability in FLs}

As a major protein of chicken egg white, OVA has an apparent molecular weight of 40, 45, 63, and $72 \mathrm{kDa}$. Both giant molecules (high molecular weight products) that could not enter the large-pore spacer gel and small molecules from OVA were observed by SDS-PAGE. In SDS-PAGE under nonreducing conditions, the OVA is visualized as the two distinctive bands $40 \mathrm{kDa}$ and $45 \mathrm{kDa}$. These are the specific bands for the identification of OVA protein, the molecular weight of which is approximately $43 \mathrm{kDa}$. This indicated OVA was encapsulated well in liposomes, and was kept active and stable in the formulations (Figure 1D).

\section{In vitro release of OVA from FLs}

The in vitro release profiles of OVA from the two types of FLs were also determined. It was found that $>85 \%$ OVA loaded onto liposome 1 was detected in the release medium within 1 hour, whereas OVA incorporated in liposome 2 showed a slower release profile with OVA contents in the release medium reaching $85 \%$ after 5 hours (Figure 2).

\section{Transcutaneous delivery of OVA-FLs in vivo}

In Figure 3, it can be seen that in the skin samples in which fluorescent liposomes were applied, intense fluorescence from the FITC label (green) could be observed with the green fluorescence detector. The liposome formulation immersed with FITC-OVA solution formed a concentrated antigen layer on its surface, an FITC label on the surface of the skin as well as along the hair follicle (Figure 3Bii), and sweat glands (Figure 3Cii)
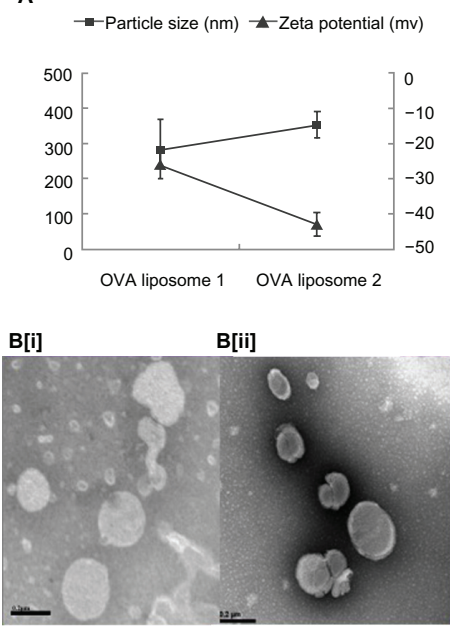

C [i]

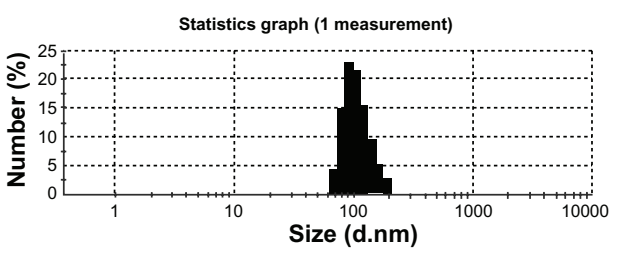

$\square$ Mean with $+l-1$ standard deviation error bar

C [ii]

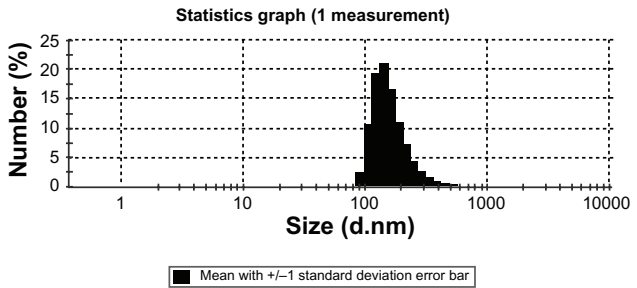

$\mathrm{D}[\mathrm{i}]$

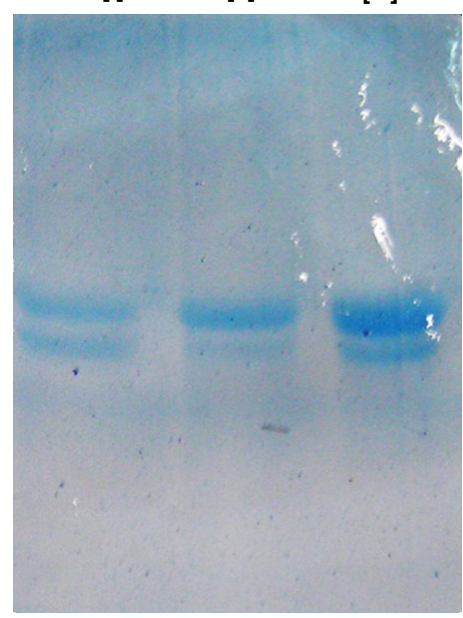

Figure I Characteristics of the ovalbumin (OVA)-loaded liposome I and 2. (A) Particle sizes and zeta potentials; (B) size distribution of the liposomes as determined by Malvern Zetamaster ([i]: liposome I; [ii] liposome 2). (C) Vesicle morphology of the liposomes under transmission electron microscopy ([i]: liposome I; [ii] liposome 2). (D) Sodium dodecyl sulfate polyacrylamide gel electrophoresis gel displaying the OVA encapsulated in liposome I and liposome 2 after loading process. Lane I: OVA standard; lane II: OVA loaded in liposome I; lane III: OVA loaded in liposome 2. 


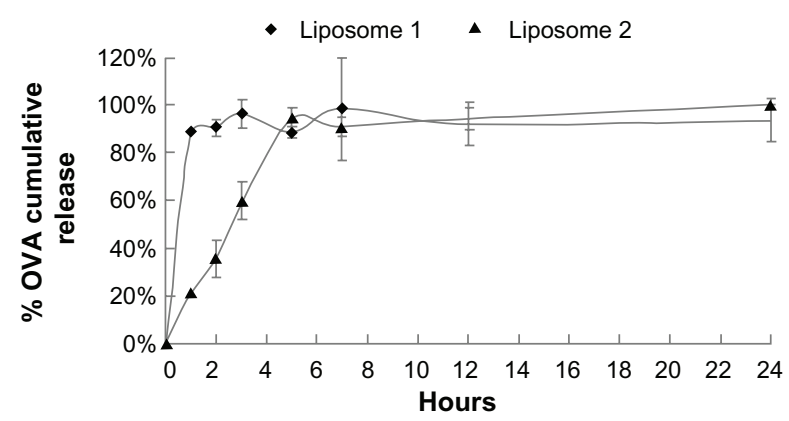

Figure 2 In vitro ovalbumin (OVA) release from the liposomes into phosphatebuffered saline $\left(37^{\circ} \mathrm{C}\right)$. Liposome I: prepared by film-dispersion method; liposome 2 : prepared by reverse-evaporation method.

inside the skin could clearly be observed. Intense fluorescence was observed at the skin mesenchymal and (hair) follicle sites after topical application of the FITC-OVA-loaded liposomes onto the skin. It is postulated that hair follicles are the major route for crossover of liposome 1. In contrast, sweat glands were the main channel for liposome 2 to pass through.

\section{Transcutaneous immunization of OVA-FLs in vivo}

Here, the in vivo efficiency of OVA-loaded liposomes in activating the transcutaneous immunization after topical application was investigated. Both FLs elicited high immune response, while liposome 1 seemed to possess a stronger efficiency in producing titer than liposome 2 . The filmdispersion method is superior for the liposome preparation for application in transcutaneous immunization (Figure 4C); therefore, liposome 1 was selected for the following in vivo study. In this study, imiquimod was tested for its efficiency in transcutaneous immunization enhancement. As can be seen in Figure 4A, compared with OVA-loaded liposome alone, OVA-loaded liposomes in combination with imiquimod achieved significantly higher levels of anti-OVA $\operatorname{IgG}(P<0.05)$ at week 8 . In another preliminary study, the removal of stratum corneum was found to enhance the antiOVA IgG levels elicited by OVA solution, so the authors

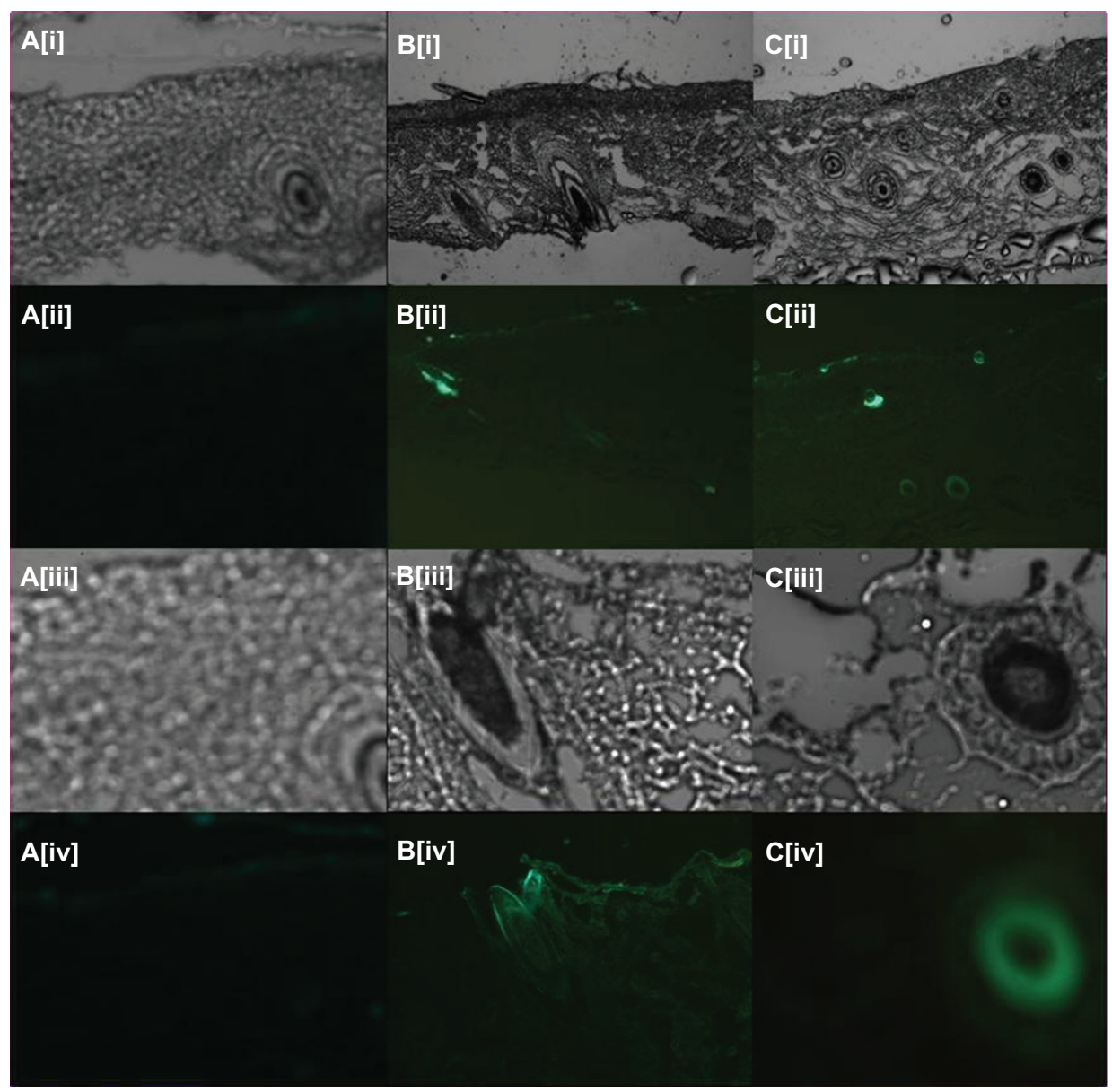

Figure 3 Fluorescent scanning microscopy images of skin samples from mice in which fluorescent ovalbumin (OVA) flexible liposomes had been applied transcutaneously: (Ai-Ci and Aiii-Ciii) images under visible light; (Aii-Cii and Aiv-Civ) images under fluorescence. Group A: OVA-loaded liposomes (blank control); Group B: FITC-OVAloaded liposome by reverse-evaporation method; Group C: FITC-OVA-loaded liposome by film-dispersion method. Notes: $(\mathbf{A i}-\mathbf{C i i})$ are shown at $\times 5$ magnification; (Aiii-Civ) are shown at $\times 20$ magnification. 
A

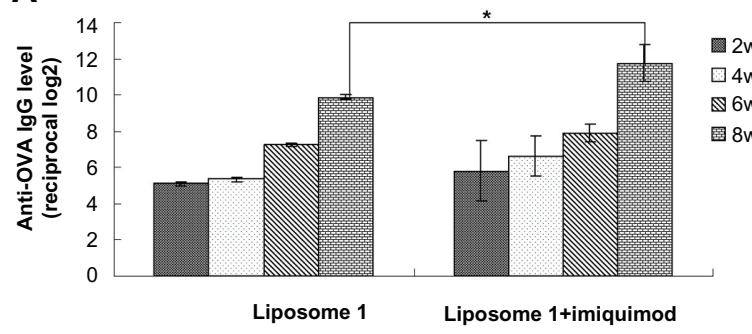

B

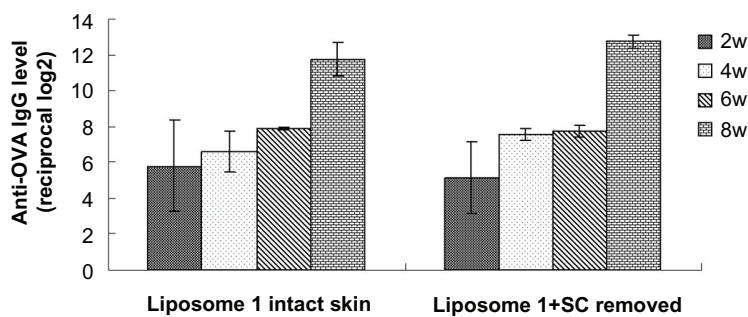

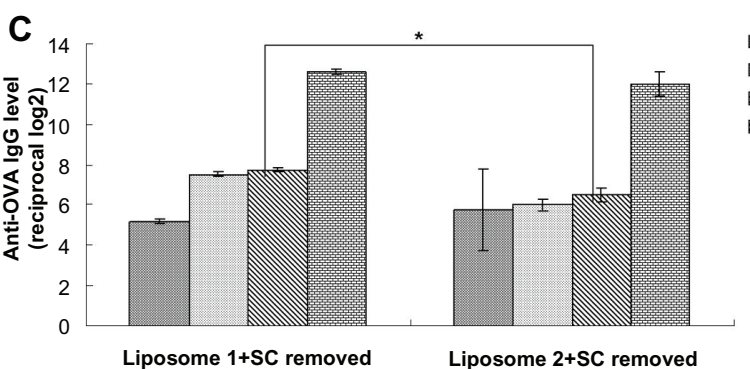

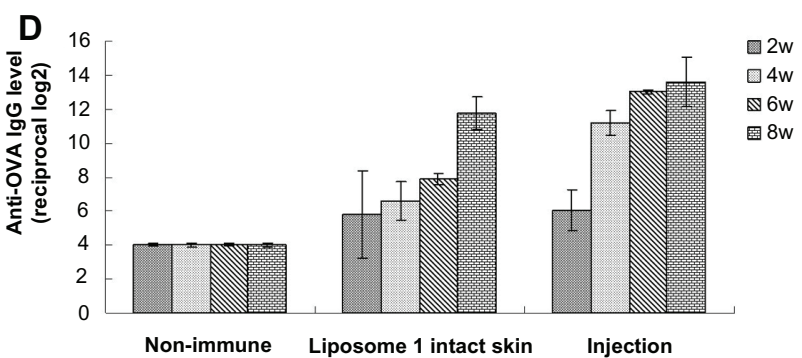

Figure 4 Anti-OVA IgG responses obtained with liposome formulations by transcutaneous immunization. (A) Liposome I (prepared by film-dispersion method) with or without adjuvant imiquimod transcutaneously. (B) Liposome I (prepared by film-dispersion method) applied on intact skin or stratum corneum pretreated skin transcutaneously, imiquimod as an adjuvant. (C) liposome I (prepared by film-dispersion method) and liposome 2 applied transcutaneously stratum corneum pretreated skin with the adjuvant imiquimod. (D) Liposome I (prepared by film-dispersion method) applied transcutaneously in the intact skin, with the adjuvant imiquimod; injection represents the OVA solution subcutaneously injected as a positive control.

Notes: Bars indicate standard deviation $(n=3)$. Statistical significance was evaluated by one-way ANOVA $(* P<0.05)$.

Abbreviations: OVA, ovalbumin; IgG, immunoglobulin G; ANOVA, analysis of variance.

further investigated whether the disruption of skin barrier would increase the transcutaneous immunization efficiency of the OVA-loaded liposome (data not shown). However, from Figure 4B, it can been seen that there was no significant difference between the anti-OVA IgG levels of the intact skin group and the stratum corneum pretreated group. Besides, the efficiency of both liposome 1 and liposome 2 was tested on the stratum corneum pretreated skin, and liposome 1 represented a significantly higher titer at the 6-week time point (Figure 4C). Based on the above results, the transcutaneous immunization effect of the OVA-loaded liposome 1 without any pretreatment of the skin was investigated. In brief, the BALB/c mice were vaccinated by either the application of nonimmune group (blank control) or liposome 1 applied onto the abdominal skin and the injection of OVA solution subcutaneously (positive control). The serum anti-OVA IgG antibody levels were then determined. By applying FL 1 on intact skin, with imiquimod as an adjuvant, strong transcutaneous immunizations were achieved (Figure 4D).

\section{Discussion}

Nanoscopic therapeutic systems that incorporate biomacromolecules, such as protein and peptides, are emerging as the next generation of nanomedicine, aiming to improve therapeutic efficacy of biomacromolecular drugs. The delivery of peptides and proteins intended for therapeutic purposes poses unique difficulties in their encapsulation, especially because most of them possess a significant hydrophobic component and thus show a slow tendency to adsorb onto surfaces. Such adsorptive behavior can lead to distinct losses in the amount of macromolecule available for delivery. ${ }^{25}$ Furthermore, the amount of peptide or protein to be incorporated within the system is dependent on the physicochemical properties of the peptide/protein and the production procedures. ${ }^{26}$ The design of novel formulations, especially lipid-based nanoscale carriers, seems promising for the disruption of stratum corneum through the nano-bio interaction of colloidal carrier with skin lipids, protection of proteins and peptides from the external environment, and maintenance of long-term activity. These properties are conducive to the application of transcutaneous vaccines. Transcutaneous immunization by the application of a simple vehicle containing antigen and adjuvant appears to be a realistic objective. However, the development of novel nanoscale systems for transcutaneous immunization is still limited by the low efficiency in eliciting robust immune response. Apart from the physical techniques to destroy the stratum corneum barrier, ultradeformable nanocarriers also evoke considerable interest in transcutaneous immunization, which may overcome the skin permeability hindrance and deliver the antigen to the immunologically active cells of skin and draining lymph nodes to raise immune response. Liposomes represent a promising carrier system for delivery 
of drugs and biologically active molecules. The lipid-based vesicles of liposomes, especially elastic liposomes, may change the bioactive permeation kinetics due to an impaired barrier function of the stratum corneum, which is helpful for skin penetration. FL generally contains phosphatidylcholine and a surfactant, and consists of at least one inner aqueous compartment surrounded by a lipid bilayer, which can carry a remarkable amount of the applied lipid mass into the skin. Its flexible bilayer can mix with stratum corneum lipids, enhance the drug disposition in skin, and disorder the barrier function. ${ }^{24,27,28}$ In our study, the flexibility of the drug-loaded liposome in enhanced skin distribution was demonstrated in comparison with CLs and solution (Table 1).

In this study, using optimized reverse-phase evaporation and film-dispersion methods, FLs with well-encapsulated OVA were prepared. SDS-PAGE results demonstrated that the OVA incorporated in the FLs were kept bioactive..$^{29,30}$ The in vitro release profiles indicated that OVA were mainly released from the FLs within 1-5 hours. Penetration mechanism study demonstrated that the OVA-FLs could gain access into the skin through the gap of skin appendages, for example, hair follicles and sweat glands. The transcutaneous immune responses induced in mice upon the application of OVA loaded FL were also examined. However, the large sizes of proteins and viral particles makes it difficult for them to penetrate through the stratum corneum, so striping the stratum corneum out of the skin to facilitate the skin penetration of proteins is therefore a strategy widely investigated for transcutaneous immunization enhancement. The penetration efficacy of the OVA-FLs through the skin with or without stratum corneum was compared. In this study, no significant difference between the anti-OVA IgG levels of the intact skin group and the stratum corneum pretreated group was observed, which confirmed that the FL system can overcome the skin barrier, increase the transcutaneous permeation of antigen, and elicit strong immune responses. The underlying mechanism for the efficiency of FLs for transcutaneous antigen delivery may be: (1) the encapsulation within such particulate delivery systems, which can protect peptides and proteins from proteolytic enzymes; (2) the stability of the protein, which resulted in the enhanced bioavailability and the improved release rate; (3) the sufficiently small size of the FL particles $(<1 \mu \mathrm{m})$, which promoted their passage across the skin through skin adhesion and particle internalization; (4) or the facilitated presence of antigen to APCs upon the enhanced elasticity of FL.

Additionally, it is known that adjuvants used in immunology are agents that may stimulate the immune system and increase the body response to a vaccine, without causing any specific antigenic effects themselves. Currently, adjuvants demonstrated with efficacy in immunization can be divided into bacterial exotoxins (subunits of the toxins are responsible for different cytokine expression from restimulated lymphocytes) and Toll-like receptor (TLR) ligands (TLRs are important signal molecules when cells sense danger) ${ }^{31-33}$ Langerhans cells express bacteria-sensing TLRs, TLR1, TLR2, TLR5, TLR6, and TLR9. In this study, imiquimod, a synthetic TLR7 ligand, which belongs to a class of immune-response modifiers, was identified as an effective adjuvant for transcutaneous immunization and enhanced the vaccination effect of OVA-FL vaccine in contrast to that of OVA-FLs alone. One of the underlying mechanisms may be that imiquimod can activate skin immune cells through TLR7 and TLR8 and induce the migration of Langerhans cells. ${ }^{34,35}$

In summary, results gained in the present study indicate that FLs can significantly improve the stability, drug entrapment, transcutaneous delivery, and the release of OVA proteins from carriers. FLs were found to be able to increase the penetration of protein through stratum corneum and retain it in the epidermis and the dermis layers, due to FLs' flexibility and its similar structure to the stratum corneum. In vivo, OVA-FLs could enter the skin through skin appendages like follicles. ${ }^{36}$ In the investigation of the immunization effects on mice, OVA-FLs exhibited a stronger immunization compared with that induced by OVA solution upon transcutaneous absorption through intact skin. It was also interesting to find that the co-administration of imiquimod can significantly increase the IgG levels induced by OVA-FLs. In future, we can expect an increasing number of therapeutic molecules such as proteins, oligonucleotides, and DNA to be carried by nanoscaled carriers as vaccine for immunization and gene therapy.

\section{Conclusion}

This study has convincingly shown the potential of FLs as a promising carrier, as well as the efficiency of the imiquimod as a novel adjuvant for transcutaneous antigen delivery, providing strong evidence for their application in transcutaneous immunization. However, in considering the future use of FLs for peptide or protein delivery, it is essential that their biosafety be studied in more intensive and elaborate trials. Particularly, a prospective in vivo tolerance evaluation in humans and/or animals should be undertaken.

\section{Disclosure}

The authors declare no conflicts of interest in this work. 


\section{References}

1. Miller MA, Pisani E. The cost of unsafe injections. Bull World Health Organ. 1999;77(10):808-811.

2. Prausnitz MR, Langer R. Transdermal drug delivery. Nat Biotechnol. 2008;26(11):1261-1268.

3. Li N, Peng LH, Chen X, Nakagawa S, Gao JQ. Transcutaneous vaccines: novel advances in technology and delivery for overcoming the barriers. Vaccine. 2011;29(37):6179-6190.

4. Peachman KK, Rao MA. Immunization with DNA through the skin. Methods. 2003;31(3):232-242.

5. Bal SM, Ding Z, van Riet E, Jiskoot W, Bouwstra JA. Advances in transcutaneous vaccine delivery: do all ways lead to Rome? J Control Release. 2010;148(3):266-282.

6. Glenn GM, Scharton-Kersten T, Alving CR. Advances in vaccine delivery: transcutaneous immunisation. Expert Opin Investig Drugs. 1999;8(6):797-805.

7. Gupta PN, Mishra V, Rawat A, et al. Non-invasive vaccine delivery in transfersomes, niosomes and liposomes: a comparative study. Int $J$ Pharm. 2005;293(1-2):73-82.

8. Ishii Y, Nakae T, Sakamoto F, et al. A transcutaneous vaccination system using a hydrogel patch for viral and bacterial infection. $J$ Control Release. 2008;131(2):113-120.

9. Matsuo K, Koizumi H, Akashi M, et al. Intranasal immunization with poly $(\gamma$-glutamic acid) nanoparticles entrapping antigenic proteins can induce potent tumor immunity. J Control Release. 2011;152(2): 310-316.

10. Yoshikawa T, Imazu S, Gao JQ, et al. Non-methylated CpG motif packaged into fusogenic liposomes enhance antigen-specific immunity in mice. DNA vaccine. Biol Pharm Bull. 2006;29(1):105-109.

11. Yoshikawa T, Okada N, Tsujino M, et al. Vaccine efficacy of fusogenic liposomes containing tumor cell-lysate against murine B16BL6 melanoma. Biol Pharm Bull. 2006;29(1):100-104.

12. Sugita T, Yoshikawa T, Gao JQ, et al. Fusogenic liposome can be used as an effective vaccine carrier for peptide vaccination to induce cytotoxic T lymphocyte (CTL) response. Biol Pharm Bull. 2005;28(1):192-193.

13. Yoshikawa T, Imazu S, Gao JQ, et al. Augmentation of antigen-specific immune responses using DNA-fusogenic liposome vaccine. Biochem Biophys Res Commun. 2004;325(2):500-505.

14. Matsuo K, Ishii Y, Quan YS, et al. Transcutaneous vaccination using a hydrogel patch induces effective immune responses to tetanus and diphtheria toxoid in hairless rat. J Control Release. 2011;149(1):15-20.

15. Yoshikawa T, Okada N, Oda A, et al. Nanoparticles built by selfassembly of amphiphilic gamma-PGA can deliver antigens to antigenpresenting cells with high efficiency: a new tumor-vaccine carrier for eliciting effector T cells. Vaccine. 2008;26(10):1303-1313.

16. Yoshikawa T, Okada N, Oda A, et al. Development of amphiphilic gamma-PGA-nanoparticle based tumor vaccine: potential of the nanoparticulate cytosolic protein delivery carrier. Biochem Biophys Res Commun. 2008;366(2):408-413.

17. Matsuo K, Yoshikawa T, Oda A, et al. Efficient generation of antigenspecific cellular immunity by vaccination with poly(gamma-glutamic acid) nanoparticles entrapping endoplasmic reticulum-targeted peptides. Biochem Biophys Res Commun. 2007;362(4):1069-1072.

18. Ramprasad MP, Amini A, Kararli T, Katre NV. The sustained granulopoietic effect of progenipoietin encapsulated in multivesicular liposomes. Int J Pharm. 2003;261(1-2):93-103.
19. Segota S, Tezak D. Spontaneous formation of vesicles. Adv Colloid Interface Sci. 2006;121(1-3):51-75.

20. Badiee A, Jaafari MR, Khamesipour A. Leishmania major: Immune response in BALB/c mice immunized with stress-inducible protein 1 encapsulated in liposomes. Exp Parasitol. 2007;115(2):127-134.

21. Xie Y, Ye L, Zhang X, et al. Transport of nerve growth factor encapsulated into liposomes across the blood-brain barrier: In vitro and in vivo studies. J Control Release. 2005;105(1-2):106-119.

22. García-Santana MA, Duconge J, Sarmiento ME, et al. Biodistribution of liposome-entrapped human gamma-globulin. Biopharm Drug Dispos. 2006;27(6):275-283.

23. Mishra D, Dubey V, Asthana A, Saraf DK, Jain NK. Elastic liposomes mediated transcutaneous immunization against Hepatitis B. Vaccine. 2006;24(22):4847-4855.

24. Wang J, Hu JH, Li F, et al. Strong cellular and humoral immune responses induced by transcutaneous immunization with $\mathrm{HBsAg}$ DNA-cationic deformable liposome complex. Exp Dermatol. 2007; 16(9):724-729.

25. Duncan MR, Lee JM, Warchol MP. Influence of surfactants upon protein/peptide adsorption to glass and polypropylene. Int J Pharm. 1995;120(2):179-188

26. Hu FQ, Hong Y, Yuan H. Preparation and characterization of solid lipid nanoparticles containing peptide. Int J Pharm. 2004;273(1-2):29-35.

27. Ding Z, Bal SM, Romeijn S, Kersten GF, Jiskoot W, Bouwstra JA. Transcutaneous immunization studies in mice using diphtheria toxoidloaded vesicle formulations and a microneedle array. Pharm Res. 2011; 28(1):145-158

28. Mishra V, Mahor S, Rawat A, et al. Development of novel fusogenic vesosomes for transcutaneous immunization. Vaccine. 2006;24(27-28): 5559-5570.

29. Guan KL, Yu W, Lin Y, Xiong Y, Zhao S. Generation of acetyllysine antibodies and affinity enrichment of acetylated peptides. Nature Protoc. 2010;5(9):1583-1595

30. Nishimura K, Ohtsuru M, Nigota K. Effect of dehydroascorbic acid on ovalbumin. J Agri Food Chem. 1989;37(6):1539-1543.

31. Williams NA. Immune modulation by the cholera-like enterotoxin B-subunits: from adjuvant to immunotherapeutic. Int J Med Microbiol. 2000;290(4-5):447-453.

32. Manning BM, Enioutina EY, Visic DM, Knudson AD, Daynes RA. CpG DNA functions as an effective adjuvant for the induction of immune responses in aged mice. Exp Gerontol. 2001;37(1):107-126.

33. Seya T, Akazawa T, Tsujita T, Matsumoto M. Role of Toll-like receptors in adjuvant-augmented immune therapies. Evid Based Complement Alternat Med. 2006;3(1):31-38

34. Shackleton M, Davis ID, Hopkins W, et al. The impact of imiquimod, a Toll-like receptor-7 ligand (TLR7 L), on the immunogenicity of melanoma peptide vaccination with adjuvant Flt3 ligand. Cancer Immun. 2004;4:9.

35. Warger T, Rechtsteiner G, Schmid B, Osterloh P, Schild H, Radsak MP. Transcutaneous immunization with imiquimod is amplified by CD40 ligation and results in sustained cytotoxic T-lymphocyte activation and tumor protection. Clin Rev Allergy Immunol. 2007;32(1):57-66.

36. Mahe B, Vogt A, Liard C, et al. Nanoparticle-based targeting of vaccine compounds to skin antigen-presenting cells by hair follicles and their transport in mice. J Invest Dermatol. 2009;129(5):1156-1164.
International Journal of Nanomedicine

\section{Publish your work in this journal}

The International Journal of Nanomedicine is an international, peerreviewed journal focusing on the application of nanotechnology in diagnostics, therapeutics, and drug delivery systems throughout the biomedical field. This journal is indexed on PubMed Central, MedLine, CAS, SciSearch $\AA$, Current Contents $\AA /$ Clinical Medicine,

\section{Dovepress}

Journal Citation Reports/Science Edition, EMBase, Scopus and the Elsevier Bibliographic databases. The manuscript management system is completely online and includes a very quick and fair peer-review system, which is all easy to use. Visit http://www.dovepress.com/ testimonials.php to read real quotes from published authors. 